\title{
Research on Parallel Combinatory Spread Spectrum Communication System with Adaptive Variable-Rate Data Transmission
}

\author{
Beiming $\mathrm{Li}^{\mathrm{a}}$, Hongyang Shi ${ }^{\mathrm{b}}$, Yixin $\mathrm{Xu}^{\mathrm{c}}$ and $\mathrm{Xue} \mathrm{Wei}^{\mathrm{d}}$
}

School of Information and Communication Engineering, Harbin Engineering University, Harbin 150001, China.

a libeiming@hrbeu.edu.cn

Keywords: parallel combinatory spread spectrum; channel estimation; variable rate.

\begin{abstract}
This paper presents a novel parallel combinatory spread spectrum (PC/SS) communication system which combining with channel estimation technology. It can be used to realize adaptive variable-rate data transmission. When the channel condition changes during the communication process, the number of the selected $P N$ sequences is changed through the signal-to-noise ratio (SNR) feed-backed from the channel estimation[1]. So as to realize the control the data transmission rate. And the number is denoted by $r$-value. The simulation results show that choosing large $r$-value for large SNR and choosing small $r$-value for small SNR can ensure the bit error rate below $10^{-3}$ and improve the average data transmission rate.
\end{abstract}

\section{Introduction}

With the development of mobile communication technology, parallel combinatory spread spectrum (PC/SS) technology has been applied to some of the burst communication and band limited communications which based on its high communication efficiency, high data transmission capability[1]. In this paper, we propose a new method to study the channel change at any time during the communication. In the communication process, the SNR ratio is fed back to the signal transmitter through channel estimation. The big $r$-value should be selected when the SNR ratio is big, and when the SNR ratio is small we should select the small $r$-value, which can ensure that the system BER in the following $10^{-3}$ when average data transfer rate is fast. Fig.1 shows the overall system block diagram.

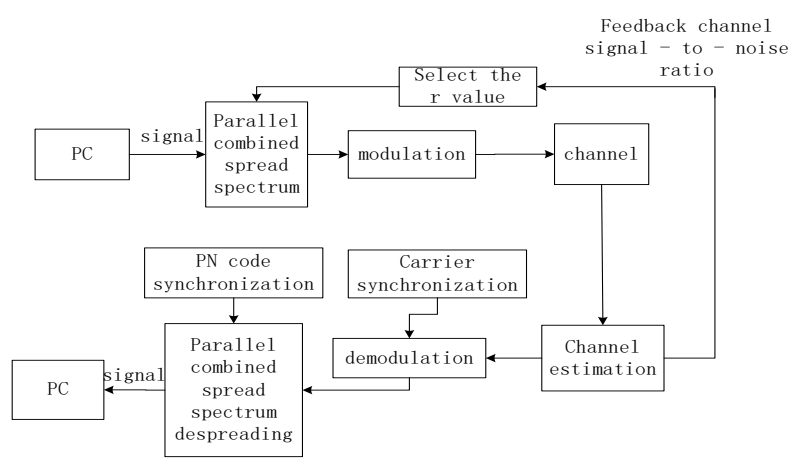

Fig. 1 The overall system block diagram

\section{System model analysis}

\subsection{Parallel combinatory spread spectrum (PC/SS)}

PC/SS communication technology is a kind of communication mode that developed on the basis of soft spread spectrum, it has relatively high information transmission capability. PC/SS can transmit information data: $K=r+\left[\log _{2}\left(C_{M}^{r}\right)\right]$, where $[X]$ is rounding to $X$. Fig2 and Fig.3 show the transmitter and receiver block diagram of PC/SS system. 
In the transmitter, $k$-bit data is sent to a data-spreading sequence mapper. The selected $P N$ sequences and a certain sequence polarity from $M$ orthogonal sequences to form a combinatory sequence. $r$ sequences are added to form a multi-value signal, and then transmitted signal after carrier

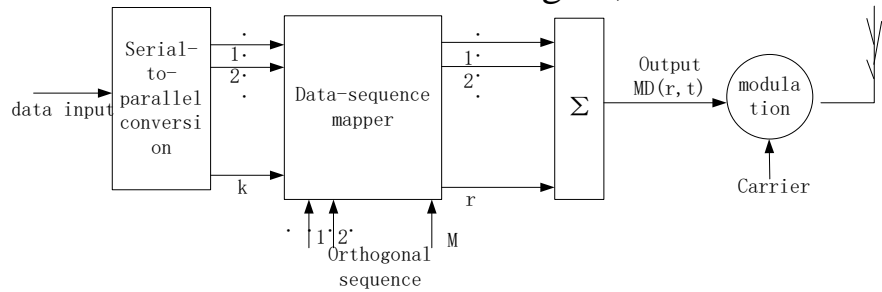

Fig.2 the transmitter block diagram of PC/SS system

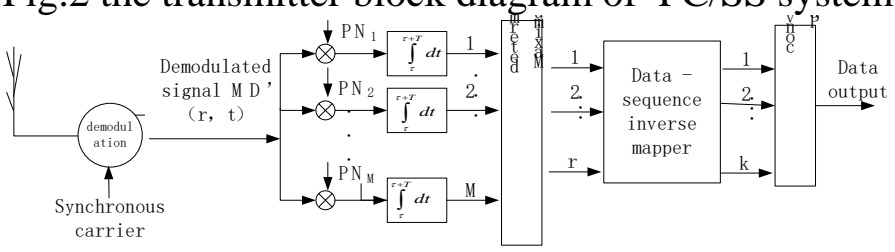

Fig.3 the receiver block diagram of PC/SS system

Modulating[2]. In the Gaussian channel, there are $M$ demodulators at the receiving end. After the carrier demodulating, the correlation calculation is performed by using $P N_{i}(t)(i=1,2, \ldots, M)$ as a despreading process. In the case of carrier and spread spectrum synchronizing, the output of ith spread spectrum demodulator is:

$$
D_{i}(\mathrm{t})=\mathrm{P} \sum_{j=1}^{r} q_{i j} \int_{\tau}^{T+\tau} P N_{i j}(\mathrm{t}-\tau) \mathrm{PN}_{i}(\mathrm{t}-\tau) \mathrm{dt}+\mathrm{N}_{i}+J_{i}(i=1,2, \cdots, M)
$$

where $\tau$ is the communication propagation delay, $i=i j$ integral is the autocorrelation of the spreading sequence, $i \neq \mathrm{i} j$ is Cross-correlation. $M$ orthogonal sequences are used:

$$
\int_{0}^{T} P N_{i j}(\mathrm{t}) P N_{i}(\mathrm{t}) \mathrm{dt}= \begin{cases}T & i j=i \\ 0 & i j \neq i\end{cases}
$$

The demodulator output is: $D_{i}=\left\{\begin{array}{cc}q_{i j} P T+N_{i}+J_{i} & i j=i \\ N_{i}+J_{i} & i j \neq i\end{array}\right.$

From the $M$ demodulator output signals selecting the spreading sequences and the polarity which correspond to the $r$ output with the largest absolute value as the transmitted combined sequence. Through the data - sequences inverse mapper, the $k$-bit data are got .It achieves spread spectrum communications.

\subsection{SNR estimation}

In this paper, the correlation estimation method is used to estimate the SNR. This method utilizes the autocorrelation characteristic of the $P N$ sequences contained in the received signal to achieve an approximate estimation of the SNR ratio[3,4]. Fig4 shows The basic block diagram.

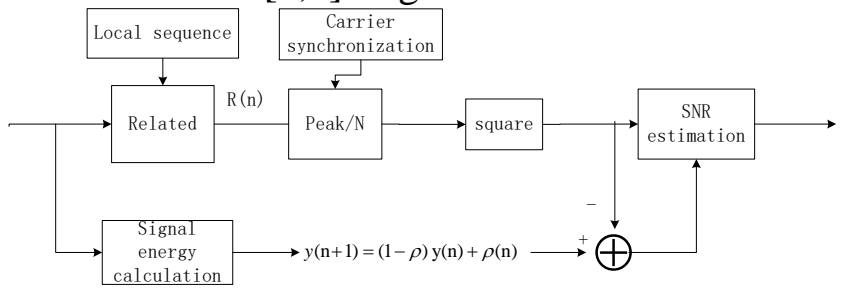

Fig.4 Known sequence autocorrelation algorithm to achieve the schematic diagram

At the receiving side, the local PN codes is used to calculate the autocorrelation with the received signal sequence. Suppose the received signal with noise is: $X(\mathrm{k})=\mathrm{a} \cdot \mathrm{S}(\mathrm{k})+\mathrm{N}(\mathrm{k}) \cdot X(k)$ and the known local sequence $S(k)$ correlation operation, you can get:

$$
R(\tau)=\mathrm{E}\left\{\left[\mathrm{aS} \mathrm{S}^{2}(\mathrm{k})+\mathrm{N}(\mathrm{k})\right][\mathrm{S}(\mathrm{k}-\tau)]\right\}
$$

According to the nature of the correlation function we can see that when $\tau=0$ can be related to the peak: $\left.R(0)=\mathrm{E}_{\left[a \mathrm{~S}^{2}\right.}(\mathrm{k})\right]+\mathrm{E}[\mathrm{S}(\mathrm{k}) \mathrm{N}(\mathrm{k})]$ Where the first term represents the signal component output by the correlator and the second term represents the noise component output by the correlator. When the sequence length is long enough, the correlator output noise is negligible. So the output of the 
correlator is $R(0) \approx \mathrm{La}$. And then you can get the signal energy $E_{s}$ through squaring it. At the same time, calculat the energy of the received signal which with noises before the correlator , and use the formula $y(\mathrm{n})=(1-\rho) \mathrm{y}(\mathrm{n})+\rho(\mathrm{n})$ to average[5]. Then the signal-to-noise ratio is calculated as follows:

$$
S N R=10 \lg \frac{E_{S}}{E-E_{S}}
$$

The $S N R$ ratio is estimated. After obtaining the $S N R$ ratio, we need to choose different $r$-values according to the different $S N R$ for $B E R$ analysis.Next, the bit error rate analysis is discussed. At the receiving end, the received signal is associated with a local spreading sequence. The result is:

$$
D_{m}(\mathrm{t})=\int_{\tau}^{T+\tau} R(\mathrm{t}) \mathrm{PN}_{m}(\mathrm{t}-\tau) \cos (\omega t+\varphi) \mathrm{dt}=V_{S}+V_{n}+V_{J}
$$

In the formula, $V_{s}$ is the signal component. When the integration function is linear, the spreading sequence is completely orthogonal. The carrier frequency and phase as well as the spreading sequence phase are synchronized:

$$
V_{S}=\left\{\begin{array}{cc}
q_{m} P T & m=i_{j} \\
0 & m \neq i_{j}
\end{array}\right.
$$

In the formula, $T$ is the time length of the spreading sequence. $V_{n}$ is zero mean and the variance is $(1 / 2) N_{0} P T$. The noise component of the double-sideband power spectral density $N_{0} / 2$ is:

$$
V_{n}=\int_{\tau}^{T+\tau} n(\mathrm{t}) \sqrt{2 P} P N_{m}(\mathrm{t}-\tau) \cos (\omega \mathrm{t}+\varphi) \mathrm{dt}
$$

$V_{J}$ is the interference component: $\quad V_{J}=\int_{\tau}^{T+\tau} J(\mathrm{t}) \sqrt{2 P} P N_{m}(\mathrm{t}-\tau) \cos (\omega \mathrm{t}+\varphi) \mathrm{dt}$

If the ith spreading sequence is one of the sequences which are the sender selecting $r$ spread spectrum sequences to send. Then the correct dispreading, the absolute value of the ith correlator output value $V_{i}$ is larger than the correlation value of the other $(M-r)$ correlator outputs. Which is $\left|V_{i}\right|>\left|V_{m}\right|,(m=1,2, \ldots, M-r)$. Therefore, the correct probability of despreading of the ith despreading correlator can be expressed as:

$$
\begin{aligned}
& P_{r_{m}}=P\left(\left|V_{i}\right|>0,\left|V_{i}\right|>\left|V_{m}\right|,(\mathrm{m}=1,2, \ldots, \mathrm{M}-\mathrm{r}) \mid V_{i}\right) \\
& =\int_{0}^{+\infty} \mathrm{p}\left(\left|V_{i}\right|>\left|V_{m}\right|\right)^{M-r} \mathrm{p}\left(V_{i} \mid\right) \mathrm{d} V_{i}
\end{aligned}
$$

Since the " 0 " and " 1 " of the transmitted data are randomly equal probability, the polarity of the selected spreading sequence is also of equal probability. It is assumed that the transmitted ith spreading sequences has positive polarity and the channel noise is Gaussian white noise. $V_{i}$ Probability density obeys the Gaussian distribution. $V_{m}$ probability density also follows the Gaussian distribution. Therefore, Equation (10) can be rewritten as follows:

$$
\begin{aligned}
& P_{r_{m}}=\int_{0}^{+\infty} \mathrm{p}\left(\left|V_{i}\right|>\left|V_{m}\right|\right)^{M-r} \mathrm{p}\left(\left|V_{i}\right|\right) \mathrm{dV}_{i} \\
& =\frac{1}{\sqrt{2 \pi}} \int_{-\sqrt{h}}^{\infty} e^{-x^{2} / 2}\left[1-\operatorname{erfc}\left(\frac{x+\sqrt{h}}{\sqrt{2}}\right)\right]^{(M-r)} d x
\end{aligned}
$$

In the formula, $h=2 k E_{b} / r N_{0}$, it is the input SNR of the ith despreading correlator. $\operatorname{erfc}(\bullet)$ is the complementary error function. The probability of the $r$ sequences outputted by the verifier are correct: $P_{r}=\left(\mathrm{P}_{r_{m}}\right)^{r}$. Since the probability of error in phase information is much lower than the probability of despreading error, it can be ignored. In this way, we can get the bit error rate formula of parallel combined spread spectrum system:

$$
P_{e_{b}}=\frac{k-r}{2 k}\left(1-P_{r}\right)=\frac{k-r}{2 k}-\frac{k-r}{2 k}\left[\frac{1}{\sqrt{2 \pi}} \int_{-\sqrt{h}}^{\infty} e^{-x^{2} / 2}\left[1-e r f c\left(\frac{x+\sqrt{h}}{\sqrt{2}}\right)\right]^{(M-r)} d x\right]^{r}
$$

As can be seen from the above two types, The error rate of the parallel combined spread spectrum communication system is mainly determined by the system parameters $M, r$ and the SNRratio $h$.

\subsection{Adaptive Variable Rate Transmission Analysis and Simulation Results}

The data transmission capability of PC/SS system is related to $M 、 r$ by formula $K=r+\left[\log _{2}\left(C_{M}^{r}\right)\right]$. In this study, the $M$ is fixed to 16 , so the data transmission capability is related to the choice of $r$ value, as shown in Table 1: 
Table $1 M=16$, choose different $\mathrm{r}$ value corresponding data transmission rate

\begin{tabular}{|c|c|c|c|c|c|}
\hline r-value & $\mathrm{r}=1$ & $\mathrm{r}=2$ & $\mathrm{r}=3$ & $\mathrm{r}=4$ & $\mathrm{r}=5$ \\
\hline Data is transmitted at one time & $5 \mathrm{bit}$ & $8 \mathrm{bit}$ & $12 \mathrm{bit}$ & $14 \mathrm{bit}$ & $16 \mathrm{bit}$ \\
\hline
\end{tabular}

Fig.7 shows that when the $r$-value is constant, the corresponding BER is smaller when the SNR is larger. And in a certain $S N R$, the error rate is smaller when selecting smaller $r$-value. The SNR which is corresponded to different $r$-values is shown in table 2,it can guarantee the BER below $10^{-3}$.

Table 2 The SNR range which is corresponded to different $r$-values

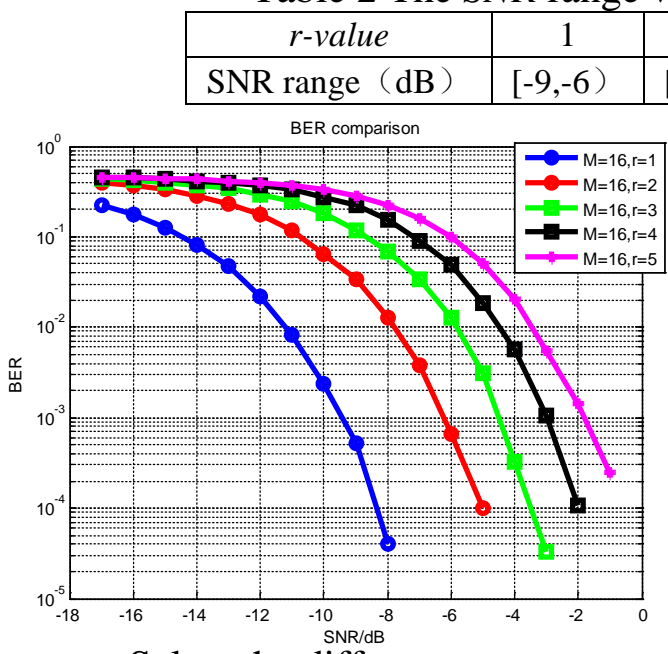

Fig.5 Select the different $r$-value

BER situation

\begin{tabular}{|c|c|c|c|}
\hline 3 & 4 & 5 & $>5$ \\
\hline$[-4,-3)$ & {$[-3,-2)$} & {$[-2,-1)$} & $>0$ \\
\hline
\end{tabular}

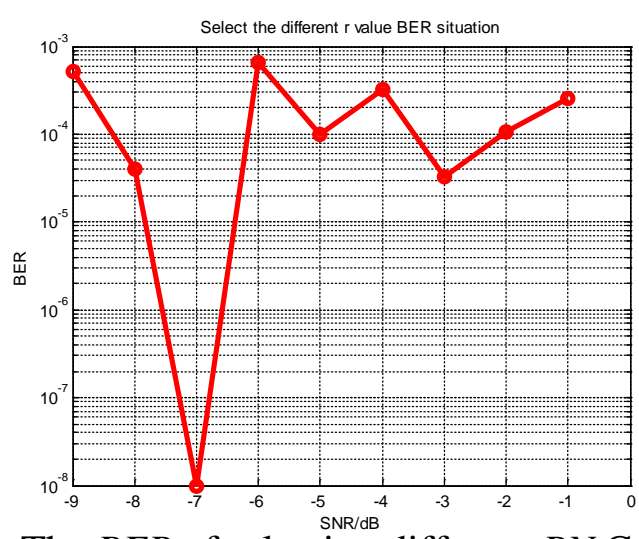

Fig.6 The BER of selecting different $P N$ Code Sequences under different $S N R$

According to the above information, we can get the signal-to-noise ratio of the current channel through SNR estimation, and choose different $r$-value to guarantee the bit error rate below $10^{-3}$ to realize the adaptive variable rate data transmission.Fig.8 shows the $B E R$ about selecting different $P N$ sequences corresponding to the different $S N R$, it is clear that the $B E R$ is lower than $10^{-3}$.

\section{Conclusions}

Combining with the channel estimation feedback technique in the parallel combinatory spread spectrum communication system, the $P N$ sequences number $r$ can be adaptively selected according to the quality of the channel. The simulation results show that when the signal-to-noise ratio is greater than $-9 \mathrm{~dB}, r$ choose 1 to 5 , the bit error rate can be guaranteed to be less than $10^{-3}$, so the system can adapt to the channel variation.

\section{Acknowledgments}

This research has been supported by International Science and Technology Cooperation Program of China (2014 DFR10240), Hei Long Jiang Postdoctoral Foundation (LBH-Z14066), Science Foundation of Heilongjiang Province QC2015075, and Fundamental Research Funds for the Central Universities GK2080260146.

\section{References}

[1] Li Beiming. Research on the key technology of Parallel combination spread spectrum communication system [D]. Harbin Engineering University, 2004.

[2] PAN Jian-Bo. Adaptive modulation in OFDM systems [D]. Beijing University of Posts and Telecommunications, 2006.

[3] Li Yinyong, Chen Jin, Zhang Yuming, Li Haicheng. Signal-to-noise ratio estimation method based on autocorrelation function [J] .Journal of Military Communications, 2011,02: 18-21.

[4] Zhang Jincheng, Peng Hua, Zhao Guoqing. Signal noise ratio estimation algorithm research [J]. Information Engineering University, 2011,05: 535-543. 
[5] Quan An. Meteor traces communication signal receiving technology and FPGA implementation [D]. Xidian University, 2010. 